\title{
Large Involuntary Forces Consistent with Plateau-Like Behavior of Human Motoneurons
}

\author{
D. F. Collins, D. Burke, and S. C. Gandevia \\ Spinal Injury Research Centre, Prince of Wales Medical Research Institute and University of New South Wales, Randwick, \\ Sydney, Australia 2031
}

When electrical stimulation is applied over human muscle, the evoked force is generally considered to be of peripheral origin. However, in relaxed humans, stimulation (1 msec pulses, 100 $\mathrm{Hz}$ ) over the muscles that plantarflex the ankle produced more than five times more force than could be accounted for by peripheral properties. This additional force was superimposed on the direct response to motor axon stimulation, produced up to $40 \%$ of the force generated during a maximal voluntary contraction, and was abolished during anesthesia of the tibial nerve proximal to the stimulation site. It therefore must have resulted from the activation of motoneurons within the spinal cord. The additional force could be initiated by stimulation of low-threshold afferents, distorted the classical relationship be- tween force and stimulus frequency, and often outlasted the stimulation. The mean firing rate of 27 soleus motor units recorded during the sustained involuntary activity after the stimulation was $5.8 \pm 0.2 \mathrm{~Hz}$. The additional force increments were not attributable to voluntary intervention because they were present in three sleeping subjects and in two subjects with lesions of the thoracic spinal cord. The phenomenon is consistent with activation of plateau potentials within motoneurons and, if so, the present findings imply that plateau potentials can make a large contribution to forces produced by the human nervous system.

Key words: human; motoneuron; muscle force; spinal cord; reflex; muscle contraction; plateau potential
The discharge of motoneurons is essential for all purposive movements. Traditionally, motoneurons are believed to summate linearly the various descending and reflex inputs that they receive (Eccles, 1957; Granit et al., 1966). However, some behavior of motoneurons does not easily fit within this view. First, there are situations in which motoneuronal discharge is excessively prolonged or inappropriately timed for the stimulus that initiated it. For example, soleus motoneurons in the cat may show prolonged self-sustained firing after activation of muscle spindle endings (Granit et al., 1957; Hultborn et al., 1975; Wada et al., 1989). In human subjects, motoneuron discharge may not be temporally locked to a large reflex input from muscle spindle afferents, despite the ability of monosynaptic pathways to phase-lock the discharge of motoneurons under other circumstances (Lang and Vallbo, 1967; Burke and Schiller, 1976). Second, animal experiments have demonstrated that motoneurons can develop plateau potentials that distort the relationship between input current and firing rate and can also produce self-sustained firing (Schwindt and Crill, 1980; Hounsgaard et al., 1988; Hounsgaard and Kiehn, 1989; Bennett et al., 1998a; Carlin et al., 2000) (for review, see Kiehn and Eken 1998; Hultborn, 1999; Hornby et al., 2000). There is indirect evidence that these mechanisms may influence motoneuron activity in conscious human subjects (Kiehn and Eken, 1997; Gorassini et al., 1998, 2000) and unrestrained animals

Received Jan. 29, 2001; revised March 13, 2001; accepted March 16, 2001.

This work was funded by the National Health and Medical Research Council of Australia (Grant number 3206). D.F.C. was supported by the National Sciences and Engineering Research Council of Canada and the Alberta Heritage Foundation for Medical Research. We are grateful to Drs. Elspeth McLachlan and James Brock for comments on this manuscript.

Correspondence should be addressed to S. C. Gandevia, Prince of Wales Medical Research Institute, Barker Street, Randwick, New South Wales, Australia 2031. E-mail: S.Gandevia@unsw.edu.au.

Copyright (C) 2001 Society for Neuroscience 0270-6474/01/214059-07\$15.00/0
(Eken and Kiehn, 1989; Gorassini et al., 1999). However, the potential "power" of any such assistance to the forces produced by human muscle has been difficult to gauge, partly because it is not possible to record intracellularly from human motoneurons in vivo and possibly because the conditions under which plateau potentials develop in experimental animals are dependent on the type and "state" of the experimental preparation (Wada et al., 1989). In humans, possible plateau activation must be inferred from more indirect measures such as single motor unit recordings (Kiehn and Eken, 1997; Gorassini et al., 1998, 2000).

Percutaneous electrical stimulation of human muscle is used extensively in both clinical and experimental settings. Such stimulation activates the muscle by directly stimulating the terminal branches of the motoneurons, and the resulting forces are thought to depend on the peripheral properties of the muscle and nerves under the stimulating electrodes. However, the stimulation will also activate sensory axons that can produce a "reflex" response in motoneurons with similarities to the tonic vibration reflex (De Gail et al., 1966; Lang and Vallbo, 1967). We have found that similar stimulation can produce surprisingly large forces and substantial distortions in the "normal" relation between stimulus frequency and the evoked force. This behavior cannot be explained by either peripheral properties of nerve and muscle or volitional drive to the motoneurons, and we propose the involvement of plateau-like properties of human motoneurons.

Some of these data have been presented in abstract form (Collins and Gandevia, 2000; Gandevia et al., 2001).

\section{MATERIALS AND METHODS}

Studies were performed on 15 able-bodied subjects (nine males, six females; age range, 24-56 years) and two paraplegic subjects with traumatic spinal cord lesions at the thoracic level (ages 39 and 43 years) studied 3 and 6 months after their accident. The procedures were approved by the local human research ethics committee. Subjects sat with 

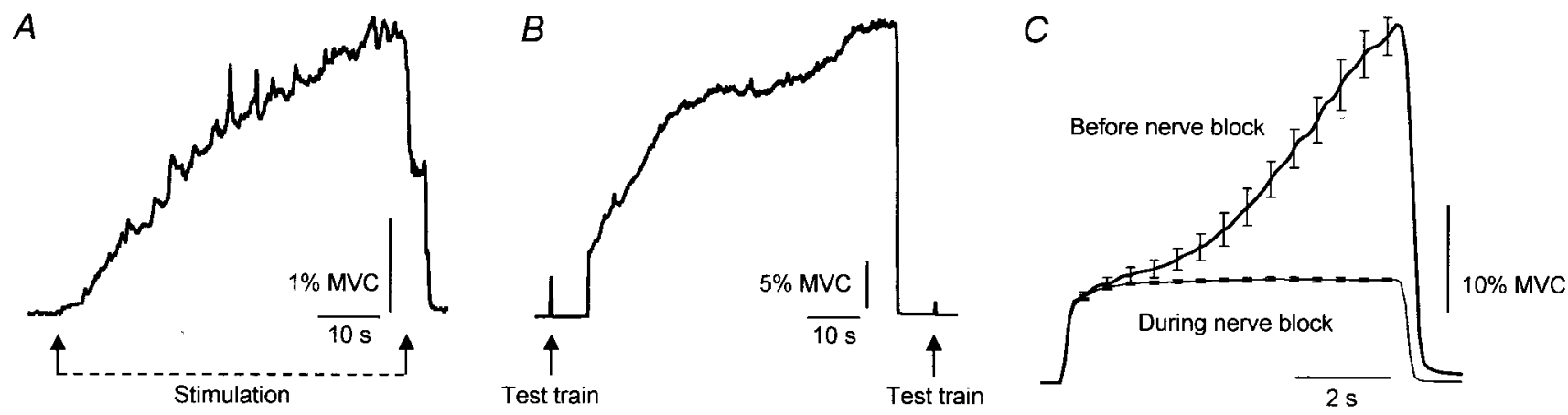

Figure 1. Forces during $100 \mathrm{~Hz}$ stimuli over the calf muscles in relaxed subjects. $A$, Force during a stimulation for 55 sec delivered at the threshold for motor axon stimulation for one subject. $B$, Force during a train lasting $60 \mathrm{sec}$ delivered above motor threshold for one subject. Note the gradual increase in force to $\sim 30 \% \mathrm{MVC}$ and the reduction in response to the test train after the prolonged stimulation. $C$, Mean responses $( \pm \mathrm{SEM})$ to five stimulus trains of $7 \mathrm{sec}$ duration in one subject before and during a complete anesthetic block of the tibial nerve. Error bars (shown at 0.5 sec intervals throughout the stimulation) are very small on the "nerve block" trace.

the hips and knees comfortably flexed and the right foot strapped to a myograph to record torque about the ankle joint (hip, knee, and ankle $\left.90-110^{\circ}\right)$. Throughout the study subjects were reminded to relax, disregard the stimulation, and were encouraged to read. Sometimes the subjects were asked to confirm that they were relaxed, and they were often asked to "relax completely", although they received no feedback about their performance during the experiment. Three subjects slept during parts of the experiment. Before the study, the maximal voluntary force (MVC) of ankle plantarflexion was measured in several attempts lasting $2-4 \mathrm{sec}$.

Electrical stimulation was applied over the calf muscles (triceps surae) via two flexible strip electrodes (10- to 18 -cm-long $\times 3.5$-cm-wide) positioned $\sim 10$ and $20 \mathrm{~cm}$ distal to the popliteal fossa. Pulses of $1 \mathrm{msec}$ duration were delivered from a stimulator that could be driven by a computer. The electrodes were positioned (with the cathode proximal) such that the stimuli produced minimal local discomfort. Stimulation intensity was adjusted based on the response to "test" trains ( 5 pulses; 1 msec duration; $100 \mathrm{~Hz}$ ) producing 2-7\% MVC. Several protocols were used in which trains of stimuli were delivered at constant intensity. (1) Trains at constant frequency: stimuli at $100 \mathrm{~Hz}$ for $\geq 7 \mathrm{sec}$ with the trains separated by $>1$ min with the 5-pulse test trains before and after the long train. (2) Trains at low frequency with one or more abrupt intermittent periods of high-frequency stimulation (e.g., $2 \mathrm{sec}$ at $25 \mathrm{~Hz}$, then $100 \mathrm{~Hz}$ for $2 \mathrm{sec}$ and finally $25 \mathrm{~Hz}$ for $3 \mathrm{sec}$ ). Control trains were delivered at 25 $\mathrm{Hz}$ only. (3) Trains with linearly changing frequencies (rising from $\sim 10-100 \mathrm{~Hz}$ over $10 \mathrm{sec}$ and declining over $10 \mathrm{sec}$ ).

In many studies surface electromyographic (EMG) activity was recorded from medial gastrocnemius (MG) and lateral gastrocnemius (LG), soleus (Sol), and tibialis anterior (TA) muscle (sampling rate 1 $\mathrm{kHz}$; bandpass $16 \mathrm{~Hz}-1 \mathrm{kHz}$ ). Torque, EMG, and stimulus parameters were recorded to disc. In seven subjects the activity of single motor units in soleus was recorded during self-sustained plantarflexion contractions via the surface EMG or a monopolar needle electrode (sampling rate 10 $\mathrm{kHz}$; bandpass $16 \mathrm{~Hz}-3 \mathrm{kHz}$ ).

In two subjects the tibial nerve was blocked in the popliteal fossa (with $12-15 \mathrm{ml}$ of $2 \%$ lidocaine with adrenaline). The nerve was initially localized with stimulation through a monopolar electrode, and the block was monitored clinically and by electrical stimulation. In one subject the block was complete, and in the other it reduced the force of maximal voluntary plantar flexion to $<10 \%$ of control and abolished the Sol $\mathrm{H}$ reflex.

\section{RESULTS}

In all subjects electrical stimulation over the calf muscles produced an additional reflex-like plantarflexion force superimposed on the force arising from stimulation of the motor axons beneath the stimulating electrodes. This "additional" force could be evoked with a range of stimulus intensities, even below motor threshold. An example of the force that developed during a long stimulus train $(100 \mathrm{~Hz})$ delivered at motor threshold is shown for one subject in Figure $1 A$. When the stimulation was applied above motor threshold, the extra force that arose in addition to the direct response to motor axon stimulation could reach $>40 \%$ MVC. Examples are shown in Figure 1, $B$ and $C$, for trains of stimuli lasting 60 and $7 \mathrm{sec}$, respectively. The additional force arose at a variable latency after the onset of the trains and could be evoked using a variety of stimulation protocols. Across eight subjects the maximal force in a prolonged train lasting $>10 \mathrm{sec}$ (as in Fig. $1 B$ ) increased 1.6-5.7 fold over the force $0.5 \mathrm{sec}$ into the train. This represented a mean increase of $21 \pm 6 \% \mathrm{MVC}$ (mean \pm SEM; range, 7-43\% MVC). Peak force occurred $44 \pm$ $11 \mathrm{sec}$ after stimulus onset (range, $8-106 \mathrm{sec}$ ). This additional force occurred even though the force due to the direct stimulation of motor axons declined. This is shown in Figure $1 B$ by the reduction in the amplitude of the response to the brief test train (five stimuli at $100 \mathrm{~Hz}$; mean reduction across the eight subjects, $56 \%$ ). This decrease presumably reflects a reduced number of stimulated motor axons (Bergmans, 1970; Vagg et al., 1998) and peripheral fatigue in the muscle fibers activated by the stimulated motor axons. Sometimes the stimulation induced a cramp in the calf muscles that could be avoided by moving the stimulating electrodes or reducing the stimulus intensity.

To exclude the possibility that these unexpected forces reflected an odd peripheral property of the stimulated motor axons and the innervated muscle fibers, we assessed responses during block of the tibial nerve in two subjects. This prevented activation of motoneurons via reflex or antidromic paths and the resulting forces could only arise from peripheral properties of the motor axons and muscle fibers beneath the stimulating electrodes. When muscles below the knee were paralyzed and "disconnected" from the CNS in this way, the additional forces associated with sustained high-frequency stimulation were absent (Fig. 1C), and the evoked forces were much more consistent and predictable.

The relationship between stimulus frequency and the evoked force was investigated with a triangular pattern of stimulation with increasing then decreasing stimulus frequencies (Fig. 2). The evoked force remained abnormally high or even increased as the stimulus frequency declined. In some instances a prominent "take off" for the extra force was identified (Fig. 2, arrow), although the stimulus frequency when this occurred was variable across subjects (between $\sim 30$ and $100 \mathrm{~Hz}$ ). This resulted in a marked distortion of the relationship between stimulus frequency and evoked force clearly evident by the hysteresis in the forcefrequency plot in Figure $2 B$. This distortion was abolished during 


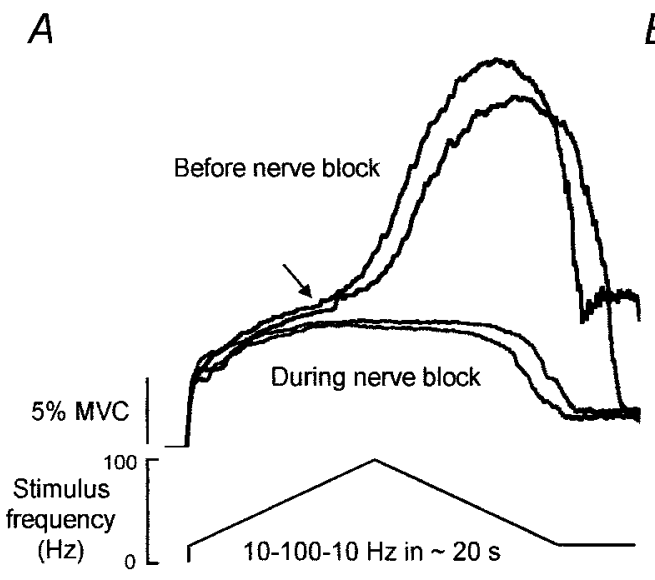

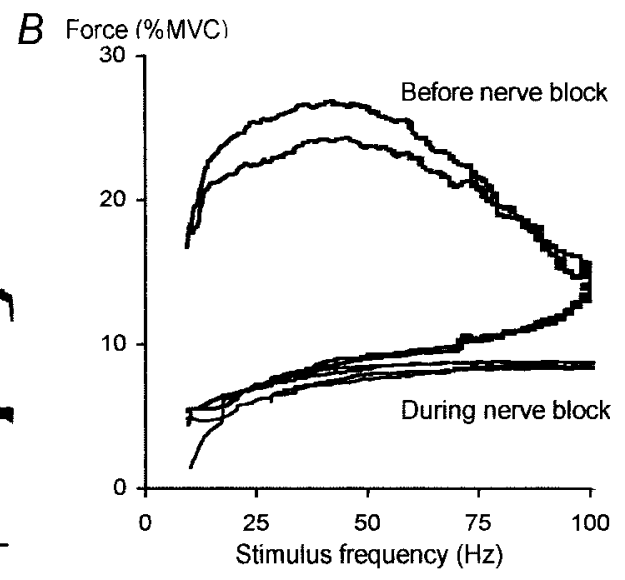

Figure 2. Responses to "triangular-shaped" changes in stimulus frequency for one subject before and during complete tibial nerve block. $A$, Responses to trains of increasing then decreasing frequency (between 10 and $100 \mathrm{~Hz}$ ) over $\sim 20 \mathrm{sec}$. The arrow marks the onset of the "extra" force. $B$, Corresponding force-frequency relationship. the tibial nerve block, thus confirming that the additional force arose from within the CNS.

To assess the force increments that arose during the highfrequency $(100 \mathrm{~Hz})$ stimulation, we delivered brief periods $(\sim 2$ sec) of such stimulation during longer trains of stimuli at $25 \mathrm{~Hz}$. These $100 \mathrm{~Hz}$ "bursts" not only increased the force much more than expected from the direct stimulation of motor axons, but when the frequency of stimulation returned to $25 \mathrm{~Hz}$, the force remained inappropriately high. This is shown for one subject in Figure $3 A$ in which there was a clear take off in the force (arrow) during the $100 \mathrm{~Hz}$ burst, after which the force remained elevated compared to the same point during the two control stimulus trains. Figure $3 B$ shows 10 superimposed responses (five control, five test) from a different subject, and the mean across the group of seven subjects is shown in Figure $3 C$. For the group, the average force $3 \mathrm{sec}$ after the end of the $100 \mathrm{~Hz}$ train was $188 \pm$ $31 \%$ of that at the same time in the control train.

Figure $4 A$ shows force and the activity of a single motor unit in Sol evoked by two successive $25 \mathrm{~Hz}$ stimulus trains with bursts at $100 \mathrm{~Hz}$ (as in Fig. 3). The initial $100 \mathrm{~Hz}$ burst (during the first stimulus train) resulted in only a small increase in force. In contrast, much more force was evoked during and after the 100 $\mathrm{Hz}$ burst of the second stimulation. Similarly, the sustained force and EMG that remained after the stimulation was turned off was much larger after the second stimulus train than the first. Figure $4 B$ shows the EMG data on an expanded time scale $(200 \mathrm{msec}$ sections) from the four points indicated by the arrows in $A$. The large vertical lines in the first four traces show the truncated stimulus artifacts from the stimulation at $25 \mathrm{~Hz}$. The first trace shows the EMG activity before the first $100 \mathrm{~Hz}$ burst (Fig. 4A, point 1 ). The only EMG activity present was time-locked to each stimulus pulse and reflected muscle activation caused by the direct stimulation of the motor axons. Part of this direct response was truncated with the stimulus artifact. The second trace is taken after the first $100 \mathrm{~Hz}$ burst (point 2) and shows the direct EMG response to the stimulation as well as the recruitment of other small motor units whose discharge was not time-locked to the stimulation. The data from the period after the second $100 \mathrm{~Hz}$ burst (Fig. 4A, point 3) show that a motor unit was recruited (at the arrow; note the reduction in gain). Trace 4 comprises the 400

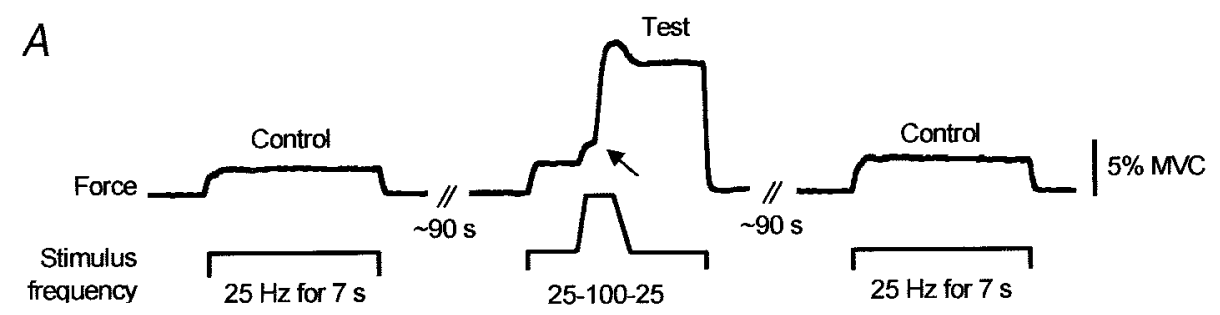

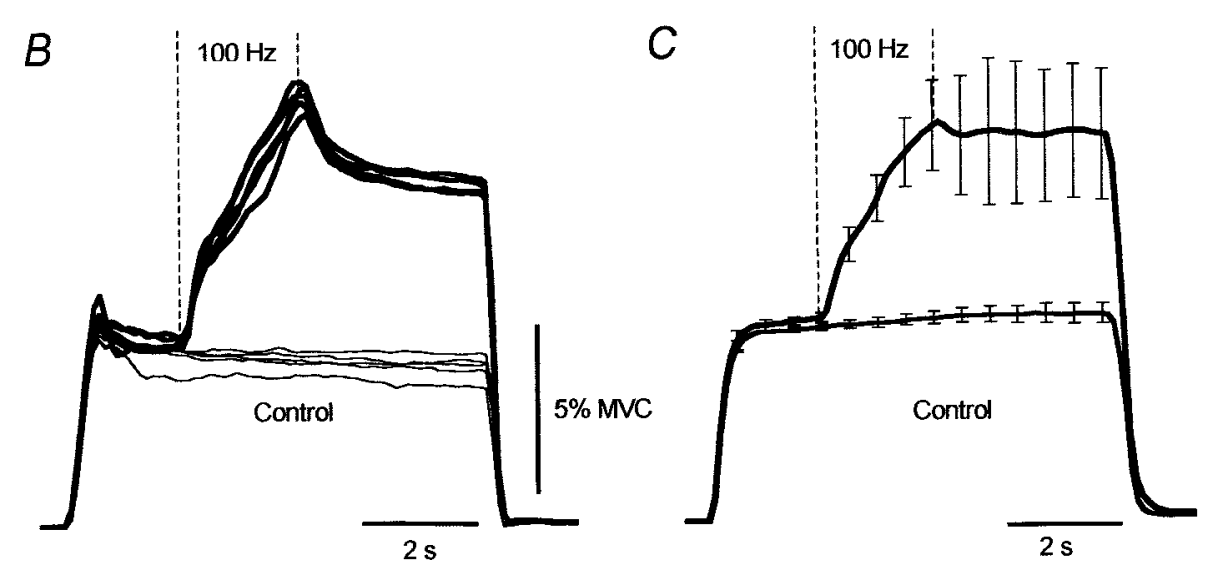

Figure 3. Forces during control trains $(25 \mathrm{~Hz})$ and during trains with additional $100 \mathrm{~Hz}$ stimulation. $A$, Force responses from one subject to three successive stimulus trains. $B$, Superimposed responses $(n=10)$ to control (thin lines) and test trains (thick lines) for one subject. $C$, Average responses for seven subjects (mean \pm SEM). Data expressed relative to the force 0.5 sec after stimulus onset in control trains. 


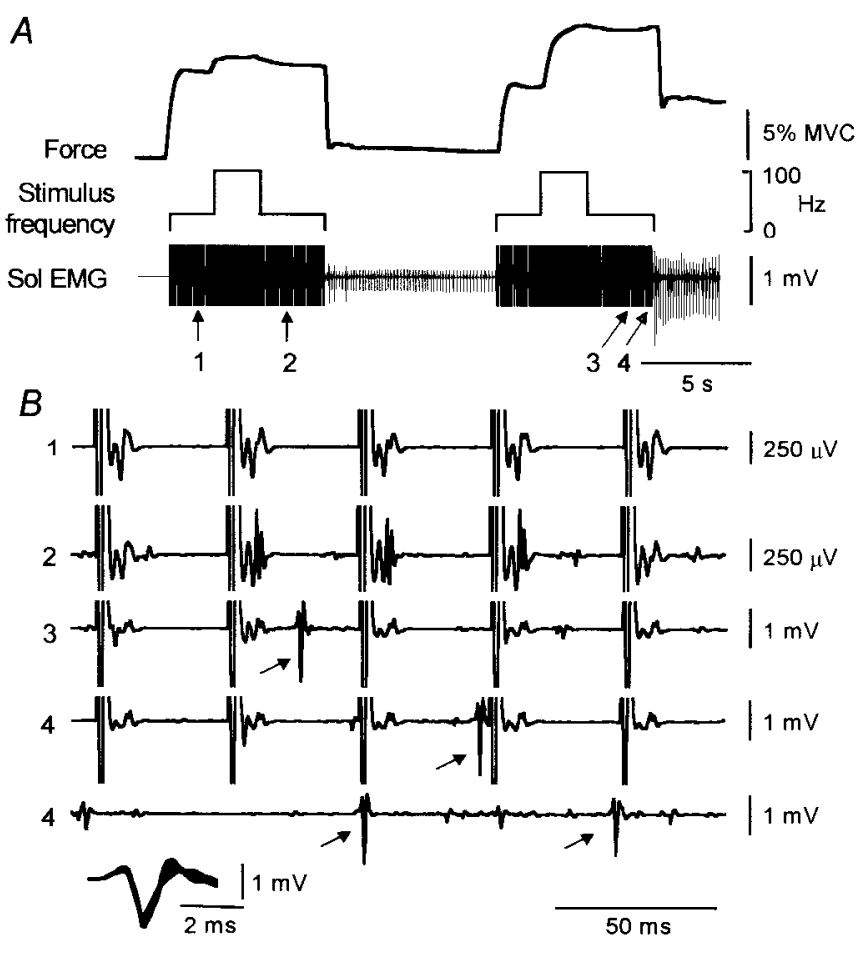

Figure 4. Force and EMG activity during two successive stimulus trains of $25 \mathrm{~Hz}$ stimulus with a $100 \mathrm{~Hz}$ burst. $A$ shows the force and EMG activity (recorded with a monopolar recording electrode) before, during, and after the stimuli. $B$ shows the unitary activity at the four points (1-4) during the stimulation shown by the arrows in $A$. Note the change in gain. The data from point 4 encompass the last five stimulus pulses and the period immediately after that. The inset at the bottom of $B$ shows the morphology of the spike highlighted by the arrows in traces 3 and 4 (6 superimposed spikes, 3 during the stimulation and 3 after).

msec period (in two parts) beginning with the last five pulses of the second stimulus train and shows that the large unit recruited at point 3 remained active throughout the remainder of the stimulus train. This unit also fired after the stimulus was turned off, initially at $\sim 10-12 \mathrm{~Hz}$, and then the frequency declined to $\sim 6-8 \mathrm{~Hz}$. The insert at the bottom of the Figure shows six superimposed spikes from the unit highlighted by the arrows in $B$ (three recorded during the stimulation and three after).

In all subjects there were instances when force was maintained after the stimulation was turned off. This sustained force could last for minutes and occurred although, when questioned, the subjects confirmed that they were "relaxed." This occurred commonly when one or more $1 \mathrm{sec}$ bursts of $100 \mathrm{~Hz}$ stimulation was delivered during $25 \mathrm{~Hz}$ trains and reflected the development of continuous EMG activity in the plantarflexor muscles, activity that had not been present before the stimulus train that evoked it (Figs. 4-6). The mean firing rate of 27 single motor units in Sol during such sustained involuntary activity was $5.8 \pm 0.2 \mathrm{~Hz}$ (coefficient of variation; $12.9 \pm 1.1 \%$ ). An example of the force and EMG activity that remained after a triangular pattern of stimulation is shown in Figure 5. In this example, brief voluntary dorsiflexion efforts failed to eliminate the sustained contraction of the plantarflexor muscles. Similar contractions (up to $10 \%$ maximum) also did not eliminate this activity in three of three subjects tested. Interestingly, if the sustained muscle contraction did not end spontaneously, a request to concentrate and relax completely usually terminated it, although when first asked subjects would typically state that they were relaxed. Figure 5 shows that the command to relax completely was not associated with activation of the antagonist muscles. No examples of sustained discharges after the stimulation was turned off were observed during the nerve blocks.

The additional forces were not attributable to inadvertent volitional descending drives to the motoneurons. They were recorded from subjects who were relaxed and often distracted by reading. They were also recorded in three subjects who went to sleep during the study (Fig. 6). Here force and EMG continued after a complex stimulus train in which the stimulus frequency declined slowly from $25 \mathrm{~Hz}$ after four $1 \mathrm{sec}$ bursts at $100 \mathrm{~Hz}$. In two paraplegic subjects with thoracic spinal cord lesions, the additional force responses were evident with the various protocols used to evoke them (Fig. 7), although these forces seemed smaller than in intact subjects.

\section{DISCUSSION}

Electrical stimulation over the calf muscles at innocuous intensities can evoke substantial plantarflexion force, much more than attributable to direct stimulation of the motor axons. When this developed the force could increase more than fivefold. Some of this additional force often remained, despite lowering the stimulus frequency or even cessation of the stimulation. This force occurred despite activity-dependent hypoexcitability in the stimulated axons (Bergmans, 1970; Vagg et al., 1998), and the peripheral fatigue expected in the motor units with the largest (and most

\begin{abstract}
Figure 5. Recording from a subject during and after a train of increasing then decreasing frequency (between $\sim 4$ and $100 \mathrm{~Hz}$ in $6 \mathrm{sec}$ ). Force continued to increase as the stimulus frequency declined, and sustained plantarflexion force and Sol EMG remained after stimulation. This persisted despite two brief efforts to dorsiflex the ankle but disappeared when the subject was asked to relax completely. EMG occurred in TA during the voluntary dorsiflexions but not when asked to relax completely. Stimulus artifacts have been truncated.
\end{abstract}

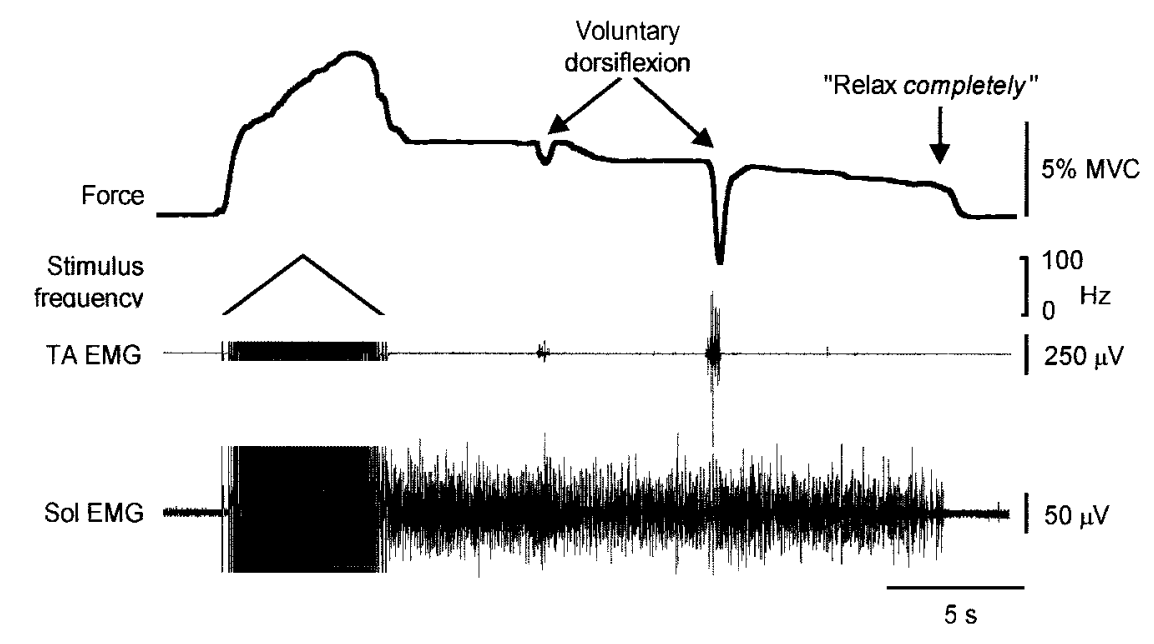




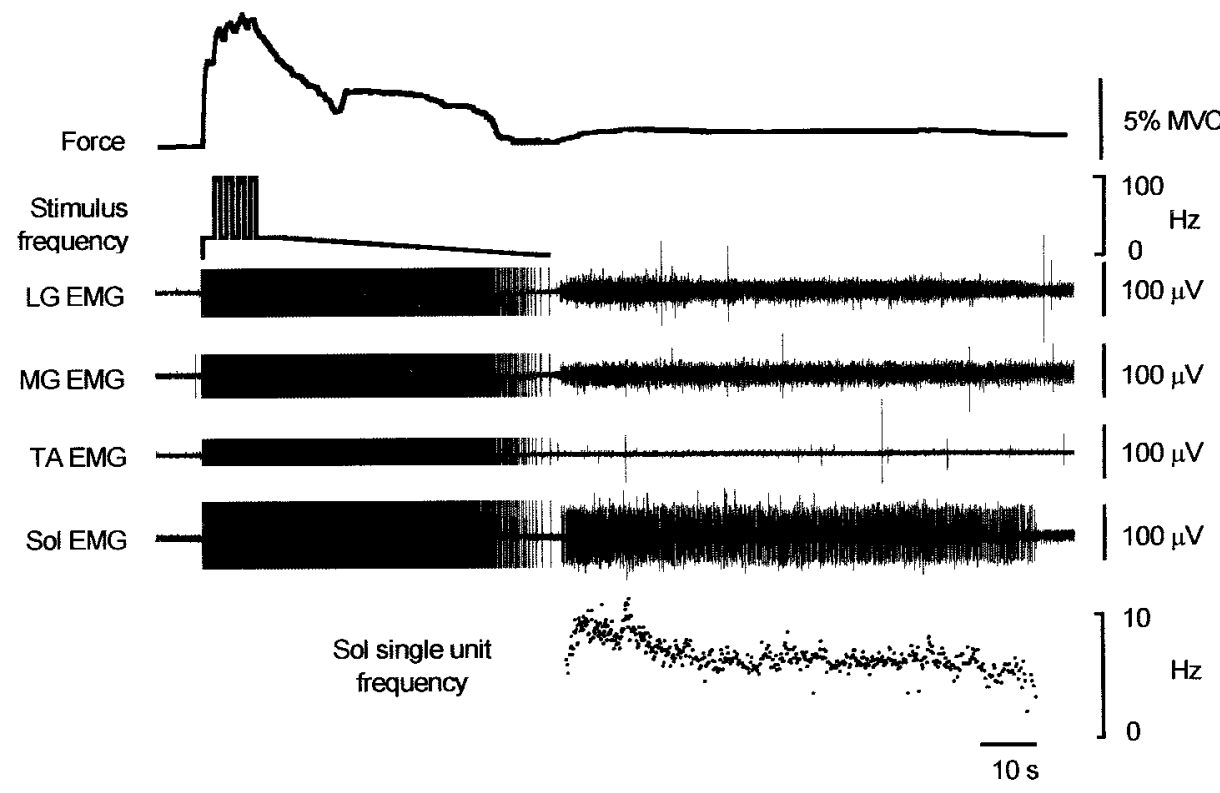

Figure 6. Recordings from a sleeping subject to a complex stimulus train that included repeated bursts of $100 \mathrm{~Hz}$ stimulation followed by a decline in frequency. Force did not decline smoothly during the slow reduction in frequency and residual force developed after the train (accompanied by EMG in Sol and MG and LG, but not TA). Stimulus artifacts have been truncated. Instantaneous discharge rate of a single motor unit in soleus is shown. easily excited) motor axons (Burke, 1981) as seen in the responses to test stimuli before and after prolonged trains (Fig. $1 B$ ).

This behavior was of central origin because it was absent when the nerve was blocked proximal to the stimulation site. During the nerve block the evoked forces could only arise from direct activation of the motor axons, and the resulting contraction forces were consistent and predictable. The presence of the unexpected force increments during stimulation at intensities below the threshold for motor axons (see also De Gail et al., 1966; Lang and Vallbo, 1967) suggest that they probably arose from stimulation of large-diameter afferents and that the antidromic activation of motoneurons was not required. Muscle rather than cutaneous afferents are likely to be important because the behavior has been observed for muscles other than the calf such as tibialis anterior (Collins and Gandevia, 2000; Gandevia et al., 2001) and biceps brachii (D. F. Collins, and S. C. Gandevia, unpublished observations) where stimulation evoked little or no cutaneous sensation. When the stimulation was applied above motor threshold, motoneurons with large-diameter axons would be preferentially activated, and antidromic volleys traveling along those axons would abolish any orthodromic action potentials arising within the spinal cord. Thus, the additional force superimposed on the direct motor response must arise from the recruitment of the smaller, more fatigue-resistant, motoneurons.

The additional forces do not reflect voluntary drive to the motoneurons because they were present in sleeping subjects and in patients with spinal cord transection. We did not document sleep stage but expect that the subjects were in non-REM sleep, a state in which voluntary intent could not have interfered. The patient data also indicate that supraspinal projections are not required, a finding consistent with recent motor unit recordings from spinal cord-injured humans (Gorassini et al., 2000) and rats (Bennett et al., 2000). However, in our experiments these additional forces could be modified by descending commands because the instruction to relax completely could abolish the sustained involuntary activity that often remained after the stimulation was turned off. Surprisingly, these self-sustained contractions of the calf muscles were not abolished by brief isometric voluntary contractions of the antagonistic muscles, demonstrating a lack of responsiveness to a particular voluntary drive that is often reciprocally organized.

The presence of these additional forces dramatically shifts the force-frequency relation of the stimulated muscle and introduces nonlinearities far greater than known for "isolated" muscle (Partridge, 1966; Binder-Macleod and Clamann, 1989). Furthermore, it makes the responses to muscle stimulation more unpredictable because the size of the extra forces varies and the onset can be triggered at different points in a stimulus train. This contrasts with the stability of the responses to the different stimulus trains during the proximal nerve block.

Given the widespread use of electrical stimulation of human muscle, it is unclear why the potency of the phenomenon reported here has not been reported previously. Our use of large surface electrodes over the muscles, long-duration pulses that favor activation of afferent axons (Veale et al., 1973; Mogyoros et al., 1996), high stimulation frequencies, and the patterns of stimulation may account for this. However, inspection of published records suggests that the additional forces to electrical stimula-
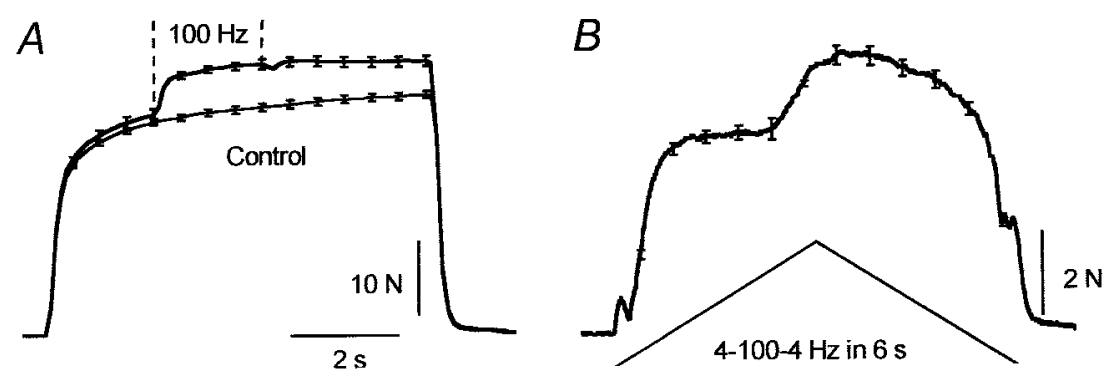

Figure 7. Recordings from two patients with spinal cord injury (mean $\pm \mathrm{SEM}, n=5$ ). $A$, Data from one subject with a complete spinal cord lesion at T12. Forces during trains of stimuli with $2 \mathrm{sec}$ at $25 \mathrm{~Hz}, 2 \mathrm{sec}$ at 100 $\mathrm{Hz}$, and then $3 \mathrm{sec}$ at $25 \mathrm{~Hz}$. For the control sequence stimulus rate was constant at $25 \mathrm{~Hz}$. B, Data from a subject with an incomplete lesion at T8. Force response to the triangular pattern of stimulation. Maximal voluntary plantarflexion force was $<10 \%$ predicted for healthy subjects. 
tion are present in some recordings (Bigland-Ritchie, 1981; Rafolt et al., 1999).

Previous studies with electrical stimulation have revealed that the discharge of human Sol motoneurons may be locked to the reflex stimulus at very low frequencies in the presence or absence of a weak voluntary contraction (as in the Hoffman or H reflex; Ashby and Zilm, 1982; Burke et al., 1984). However, in relaxed subjects the $\mathrm{H}$ reflex declines with increasing rates of stimulation (Burke and Schiller, 1976; Burke et al., 1989; Crone and Nielsen, 1989; Hultborn et al., 1996). It is often overlooked that at high stimulus frequencies this locking is absent and that the discharge of recruited motoneurons is not locked to the driving stimulus, as shown in Figure 4 (De Gail et al., 1966; Lang and Vallbo, 1967; Burke and Schiller, 1976). One explanation for the present results is that motoneurons are recruited by the high-frequency afferent bombardment and their discharge is then sustained via activation of plateau potentials within them. The motoneurons then become temporally uncoupled from the reflex inputs which, under other conditions, can drive them through largely monosynaptic reflex paths (Burke et al., 1984).

Plateau potentials leading to bistable states are generated in many central neurons (Fraser and MacVicar, 1996; Morisset and Nagy, 1999) and have been demonstrated in mammalian motoneurons (Schwindt and Crill, 1980; Hounsgaard et al., 1988; Hounsgaard and Kiehn, 1989; Bennett et al., 1998a; Lee and Heckman, 1998; Carlin et al., 2000) (for review, see Hornby et al., 2000). Several features of the present results are consistent with the involvement of plateau potentials in motoneurons. First, the additional force depended on the activation of the smaller, more fatigue-resistant motoneurons (Lee and Heckman, 1998, 2000) by high-frequency synaptic input from large-diameter afferents (Hultborn et al., 1975; Bennett et al., 1998a; Lee and Heckman, 1998); second, it often became more prominent with repeated bursts of stimulation analogous to the "wind up" phenomenon (Bennett et al., 1998b); third, it could generate self-sustained firing (Hounsgaard et al., 1988; Wada et al., 1989; Kiehn and Eken, 1997; Gorassini et al., 1998); fourth, it could often be terminated by particular "inputs" to the motoneuron pool (Hultborn et al., 1975; Lee and Heckman, 1998), and finally, motor unit discharge frequencies were similar to those in a previous human study that probably involved plateau potentials (Kiehn and Eken, 1997). Alternatively, the abnormal force increments may derive from plateau-like activity in spinal interneurons, or a "reverberating" spinal circuit (Hultborn et al., 1975), although there is currently little evidence for such a circuit.

Regardless of the underlying mechanism, stimulation applied over human muscles can recruit motoneurons synaptically, presumably according to their natural recruitment order (small to large; Henneman, 1957; Henneman et al., 1965). This may have applications for functional electrical stimulation in which muscle fatigue is often a problem caused by the preferential activation of motoneurons with large-diameter axons. The additional recruitment of the smaller motoneurons that innervate more fatigueresistant muscle fibers may be particularly useful for generating sustained contractions required for tasks such as standing. Similarly, because the stimulation activates a portion of the muscle not normally activated by direct motor axon stimulation, it may have applications in reducing the muscle atrophy resulting from disuse associated with acute or chronic injury conditions.

Plateau potentials have been invoked in other studies on human subjects to explain cramp-like behavior (Baldissera et al., 1991, 1994), self-sustained discharges (Kiehn and Eken, 1997;
Gorassini et al., 1998), and the potential discrepancy between the forces at recruitment and derecruitment of motor units (Heckman and Lee, 1999). Whereas these studies focused on EMG recordings and the properties of single motor units, our study focused on the resulting forces and suggests that these intrinsic mechanisms can generate large forces and thereby make a substantial contribution to the control of voluntary movement. Thus, present understanding of the in vivo operation of human motoneuron pools may need revision.

\section{REFERENCES}

Ashby P, Zilm D (1982) Characteristics of postsynaptic potentials produced in single human motoneurons by homonymous group 1 volleys. Exp Brain Res 47:41-48.

Baldissera F, Cavallari P, Dworzak F (1991) Cramps: a sign of motoneurone "bistability" in a human patient. Neurosci Lett 133:303-306.

Baldissera F, Cavallari P, Dworzak F (1994) Motor neuron "bistability". A pathogenetic mechanism for cramps and myokymia. Brain 117:929-939.

Bennett DJ, Hultborn H, Fedirchuk B, Gorassini M (1998a) Synaptic activation of plateaus in hindlimb motoneurons of decerebrate cats. J Neurophysiol 80:2023-2037.

Bennett DJ, Hultborn H, Fedirchuk B, Gorassini M (1998b) Short-term plasticity in hindlimb motoneurons of decerebrate cats. J Neurophysiol 80:2038-2045.

Bennett DJ, Gorassini M, Yunru L, Sanelli L (2000) Plateaus and spasticity in chronic sacral spinal rats. Plateau potentials, and rhythmic firing in motoneurons, an international conference organized by R. M. Enoka and C. J. Heckman, Boulder, CO, June. (http://www.colorado. edu/kines/BoulderMeeting.html).

Bergmans J (1970) The physiology of single human nerve fibres. Vander: University of Louvain.

Bigland-Ritchie B (1981) EMG and fatigue of human voluntary and stimulated contractions. Ciba Found Symp 82:130-156.

Binder-Macleod SA, Clamann HP (1989) Force output of cat motor units stimulated with trains of linearly varying frequency. J Neurophysiol 61:208-217.

Burke D, Schiller HH (1976) Discharge pattern of single motor units in the tonic vibration reflex of human triceps surae. J Neurol Neurosurg Psychiatry 39:729-741.

Burke D, Gandevia SC, McKeon B (1984) Monosynaptic and oligosynaptic contributions to the human ankle jerk and H-reflex. J Neurophysiol 52:435-447.

Burke D, Adams RW, Skuse NF (1989) The effects of voluntary contraction on the $\mathrm{H}$ reflex of human limb muscles. Brain 112:417-433.

Burke RE (1981) Motor units: anatomy, physiology and functional organisation. In: Handbook of Physiology (Brooks V B, ed), pp 345-421. Bethesda, MD: American Physiological Society.

Carlin KP, Jones KE, Jiang Z, Jordan LM, Brownstone RM (2000) Dendritic L-type calcium currents in mouse spinal motoneurons: implications for bistability. Eur J Neurosci 12:1635-1646.

Collins DF, Gandevia SC (2000) Afferent activation contributes to force production during electrical stimulation of human muscle. Soc Neurosci Abstr 26:2215.

Crone C, Nielsen J (1989) Methodological implications of the post activation depression of the soleus H-reflex in man. Exp Brain Res $78: 28-32$.

De Gail P, Lance JW, Neilson PD (1966) Differential effects on tonic and phasic reflex mechanisms produced by vibration of muscles in man. J Neurol Neurosurg Psychiatry 29:1-11.

Eccles JC (1957) The physiology of nerve cells. Baltimore: Johns Hopkins UP.

Eken T, Kiehn O (1989) Bistable firing properties of soleus motor units in unrestrained rats. Acta Physiol Scand 136:383-394.

Fraser DD, MacVicar BA (1996) Cholinergic-dependent plateau potential in hippocampal CA1 pyramidal neurons. J Neurosci 16:4113-4128.

Gandevia SC, Burke D, Collins DF (2001) Possible role of plateau potentials in force increments produced by electrical stimulation over human muscles. Proc Austr Neurosci Soc 12:228.

Gorassini M, Bennett DJ, Yang JF (1998) Self-sustained firing of human motor units. Neurosci Lett 247:13-16.

Gorassini M, Bennett DJ, Kiehn O, Eken T, Hultborn H (1999) Activation patterns of hindlimb motor units in the awake rat and their relation to motoneuron intrinsic properties. J Neurophysiol 82:709-717.

Gorassini M, Bennett DJ, Yang JF, Harvey P (2000) Evidence for activation of $I_{\text {PICS }}$ from firing behaviour of motor unit pairs in uninjured and spinal cord injured human subjects. Plateau potentials and rhythmic firing in motoneurons, an international conference organized by R. M. Enoka and C. J. Heckman, Boulder, CO, June. (http://www.colorado.edu/kines/BoulderMeeting.html).

Granit R, Phillips CG, Skoglund S, Steg G (1957) Differentiation of tonic 
from phasic alpha ventral horn cells by stretch, pinna and crossed extensor reflexes. J Neurophysiol 20:470-481.

Granit R, Kernell D, Lamarre Y (1966) Algebraical summation in synaptic activation of motoneurones firing within the "primary range" to injected currents. J Physiol (Lond) 187:379-399.

Heckman CJ, Lee RH (1999) Synaptic integration in bistable motoneurons. In: Progress in brain research (Binder MD, ed), pp 49-56. Amsterdam: Elsevier Science.

Henneman E (1957) Relation between size of neurons and their susceptibility to discharge. Science 126:1345-1347.

Henneman E, Somjen G, Carpenter DO (1965) Functional significance of cell size in spinal motoneurons. J Neurophysiol 28:560-580.

Hornby TG, Stauffer EK, Stuart DG (2000) Open issues on the functional role of the plateau potential in the repetitive discharge of motoneurons in experimental animals and humans. In: Sensorimotor control (Dengler R, Kossev AR, eds), Amsterdam: IOS.

Hounsgaard J, Kiehn O (1989) Serotonin-induced bistability of turtle motoneurones caused by a nifedipine-sensitive calcium plateau potential. J Physiol (Lond) 414:265-282.

Hounsgaard J, Hultborn H, Jespersen B, Kiehn O (1988) Bistability of alpha-motoneurones in the decerebrate cat and in the acute spinal cat after intravenous 5-hydroxytryptophan. J Physiol (Lond) 405:345-367.

Hultborn H (1999) Plateau potentials and their role in regulating motoneuronal firing. Prog Brain Res 123:39-48.

Hultborn H, Wigstrom H, Wangberg B (1975) Prolonged activation of soleus motoneurones following a conditioning train in soleus Ia afferents-a case for a reverberating loop? Neurosci Lett 1:147-152.

Hultborn H, Illert M, Nielsen J, Paul A, Ballegaard M, Wiese H (1996) On the mechanism of the post-activation depression of the H-reflex in human subjects. Exp Brain Res 108:450-462.

Kiehn O, Eken T (1997) Prolonged firing in motor units: evidence of plateau potentials in human motoneurons? J Neurophysiol 78:3061-3068.
Kiehn O, Eken T (1998) Functional role of plateau potentials in vertebrate motor neurons. Curr Opin Neurobiol 8:746-752.

Lang AH, Vallbo ÅB (1967) Motoneuron activation by low intensity tetanic stimulation of muscle afferents in man. Exp Neurol 18:383-391.

Lee RH, Heckman CJ (1998) Bistability in spinal motoneurons in vivo: systematic variations in rhythmic firing patterns. J Neurophysiol 80:572-582.

Lee RH, Heckman CJ (2000) Adjustable amplification of synaptic input in the dendrites of spinal motoneurons in vivo. J Neurosci 20:6734-6740.

Mogyoros I, Kiernan MC, Burke D (1996) Strength-duration properties of human peripheral nerve. Brain 119:439-447.

Morisset V, Nagy F (1999) Ionic basis for plateau potentials in deep dorsal horn neurons of the rat spinal cord. J Neurosci 19:7309-7316.

Partridge DL (1966) Signal-handling characteristics of load-moving skeletal muscle. Am J Physiol 210:1178-1191.

Rafolt D, Gallasch E, Mayr W, Lanmuller H (1999) Dynamic force responses in electrically stimulated triceps surae muscles: effects of fatigue and temperature. Artif Organs 23:436-439.

Schwindt P, Crill W (1980) Role of a persistent inward current in motoneuron bursting during spinal seizures. J Neurophysiol 43:1296-1318.

Vagg R, Mogyoros I, Kiernan MC, Burke D (1998) Activity-dependent hyperpolarization of human motor axons produced by natural activity. J Physiol (Lond) 507:919-925.

Veale JL, Mark RF, Rees S (1973) Differential sensitivity of motor and sensory fibres in human ulnar nerve. J Neurol Neurosurg Psychiatry 36:75-86.

Wada N, Nakajima Y, Homma S (1989) Long-lasting afterdischarge of alpha-motoneurons after muscle vibration or electrical stimulation of Group I afferent fibers in the anemically decerebrated cat. Neurosci Res 6:234-247. 